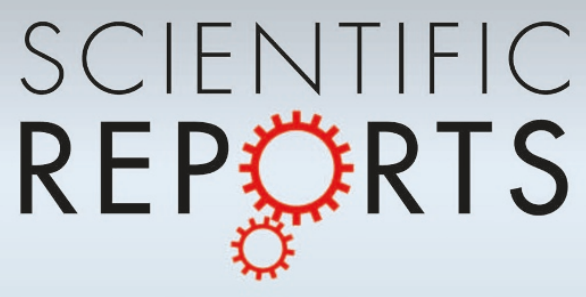

OPEN

SUBJECT AREAS:

ELECTRONIC PROPERTIES

AND MATERIALS

NONLINEAR PHENOMENA

SCANNING PROBE

MICROSCOPY

MATERIALS SCIENCE

Received

21 June 2013

Accepted

27 August 2013

Published

17 September 2013

Correspondence and requests for materials should be addressed to S.V.K. (sergei2@ornl. gov)

* These authors contributed equally to this work.

$\uparrow$ Current address: The Center for Nanophase Materials Sciences, Oak Ridge National Laboratory, Oak Ridge, TN 37831.

† Current address: School of Advanced Materials Science and

Engineering,

Sungkyunkwan

University, Suwon 440-746, South

Korea.

\section{Higher order harmonic detection for exploring nonlinear interactions with nanoscale resolution}

\author{
R. K. Vasudevan ${ }^{* \dagger} \dagger$, M. Baris Okatan ${ }^{*} \dagger$, I. Rajapaksa ${ }^{2}$, Y. Kim ${ }^{2}+$, D. Marincel ${ }^{3}$, S. Trolier-McKinstry ${ }^{3}$, \\ S. Jesse ${ }^{2}$, N. Valanoor' \& S. V. Kalinin ${ }^{2}$
}

\begin{abstract}
${ }^{1}$ School of Materials Science and Engineering, University of New South Wales, Kensington, Sydney 2052, Australia, ${ }^{2}$ The Center for Nanophase Materials Sciences, Oak Ridge National Laboratory, Oak Ridge, TN 37831 , ${ }^{3}$ Department of Materials Science and Engineering, Pennsylvania State University, University Park, Pennsylvania 16802 (USA)
\end{abstract}

Nonlinear dynamics underpin a vast array of physical phenomena ranging from interfacial motion to jamming transitions. In many cases, insight into the nonlinear behavior can be gleaned through exploration of higher order harmonics. Here, a method using band excitation scanning probe microscopy (SPM) to investigate higher order harmonics of the electromechanical response, with nanometer scale spatial resolution is presented. The technique is demonstrated by probing the first three harmonics of strain for a $\mathrm{Pb}\left(\mathrm{Zr}_{1-\mathrm{x}} \mathrm{Ti}_{\mathrm{x}}\right) \mathrm{O}_{3}(\mathrm{PZT})$ ferroelectric capacitor. It is shown that the second order harmonic response is correlated with the first harmonic response, whereas the third harmonic is not. Additionally, measurements of the second harmonic reveal significant deviations from Rayleigh-type models in the form of a much more complicated field dependence than is observed in the spatially averaged data. These results illustrate the versatility of $\mathrm{n}^{\text {th }}$ order harmonic SPM detection methods in exploring nonlinear phenomena in nanoscale materials.

$\mathrm{N}$ onlinear systems are ubiquitous in nature, ranging from optics ${ }^{1}$ to electronics to chemical reactions ${ }^{2}$. An important subset of these phenomena concerns the study of nonlinear dynamics as manifested in solids, such as the motion of interfaces in ferroic materials ${ }^{3,4}$, onset of superconductive ${ }^{5}$ and polar phases ${ }^{6}$, jamming transitions ${ }^{7}$, and electrochemical reactions $s^{8}$. These phenomena are an inherent part of technologically important materials and systems such as batteries, fuel cells, martensites, ferroics, and many more. As such, understanding sources and mechanisms of nonlinear responses and their role in emergent materials functionality is a key challenge of science.

One well known method of exploring nonlinear responses is to examine the higher order harmonics of the response of the material to an applied stimulus. For example, the use of second harmonic generation for probing crystal structures, surface states and magnetic structure ${ }^{9}$, whilst force-based scanning capacitance microscopy (which also utilizes the second harmonic) has been prevalent since the early $90^{\prime} \mathrm{s}^{10}$. Despite this recognition of the role of higher order harmonic analyses, full understanding of the nonlinear dynamics over a variety of length scales has been stymied by a lack of suitable techniques. Presently, there is a lack of understanding of how effects driven by extremely local materials phenomena (in the $\mathrm{nm}-\mu \mathrm{m}$ range) translate collectively into a much larger device-level impact.

Recently we have shown that the individual behavior of domain walls in ferroelectrics at the local level is different from that expected from the traditional Rayleigh framework ${ }^{11}$. Here, a new SPM technique using $\mathrm{n}^{\text {th }}$ order harmonic detection and band-excitation is demonstrated that allows decoupling of responses in microscale volumes. A well-characterized ferroelectric material, namely epitaxial PZT, is used as a model system. It is found that the response of the second harmonic has locally non-monotonic behavior that forms spatial clusters which were thus far "invisible" when using methods limited to collecting spatially averaged data. It is speculated that these departures arise from local frozen disorder. These studies discover a rich spectrum of emergent spatiallyinhomogeneous behaviors that may have broad implications for study of nonlinearity in other systems.

The basis for our approach rests on the fact that system responses can be approximated as linear for small stimuli. Either increasing the amplitude of the driving force or probing second and higher order harmonic responses permits exploration of nonlinear phenomena ${ }^{12,13}$. Many phenomena, such as Joule heating ${ }^{14}$, electrostriction $^{15}$, and electrostatic actuation ${ }^{10}$, rely on the second harmonic of the stimulus (e.g. electric field), whereas 
primarily linear phenomena (such as intrinsic piezoelectric coupling) have much smaller higher order harmonic terms. As a result, the decomposition of the total response into harmonic components offers a path to decoupling individual response contributions to the total signal.

Seminal work on ferroelectrics capacitors was undertaken by Turik $^{16}$ in the 1960s. Since then, research into the nonlinear properties of ferroelectric capacitor structures has been studied theoretically by Boser ${ }^{17,18}$, (developing earlier statistical physics analyses by Kronmüller ${ }^{19}$ ) and extended with experimental investigations by Damjanovic ${ }^{20-22}$ and Trolier-McKinstry et al..$^{23,24}$. Other significant experimental ${ }^{25-27}$ and theoretical ${ }^{28}$ studies on harmonic analysis have also been reported. However, these measurements have generally been performed on macroscopic systems, e.g. using top-electroded capacitor structures which sample large volumes of material. Hence, spatial resolution and therefore, the ability to correlate the response with microstructural features, have been unavailable. As a second challenge, higher order harmonics can be very small, with low susceptibility constants when compared to the linear couplings present. Finally, instrument-dominated contributions to the higher order harmonics are not uncommon.

As a model for nonlinear interaction mapping, we explore the first three harmonics of the strain response in a ferroelectric PZT capacitor. Similar capacitors have been well-studied macroscopically ${ }^{21,23,24}$, and the theoretical framework for the harmonic responses has been developed recently ${ }^{29-31}$. Furthermore, the technological applications of ferroelectric capacitors, to act as transducers ${ }^{32}$ and actuators ${ }^{33}$, necessitate a more thorough understanding of the nonlinear dynamics in these systems.

In polycrystalline ferroelectrics, the strain and polarization responses at low to mid-range driving fields often follow the phenomenological Rayleigh Law, which can be expressed as:

$$
\begin{gathered}
d_{33}=d_{\text {init }}+\alpha_{D} E \\
x(E)=\left(d_{\text {init }}+\alpha_{D} E_{0}\right) E \pm \frac{\alpha_{D}}{2}\left(E^{2}-E_{0}^{2}\right)
\end{gathered}
$$

where $\mathrm{d}_{33}$ is the piezoelectric coefficient, $x$ is the strain, $E=E_{0}$ $\sin (\omega t)$ is the applied field, $\mathrm{E}_{0}$ is the maximum field in the sweep, $\alpha_{D}$ is the irreversible Rayleigh coefficient, and $d_{\text {init }}$ is the reversible piezoelectric Rayleigh coefficient. The ' + ' refers to the reverse (decreasing) branch of the voltage sweep, and '-' refers to the forward (increasing) branch.

While the Rayleigh law has been shown to be largely valid for ferroic systems (under appropriate conditions, such as a Gaussian distribution of restoring forces acting on the domain wall ${ }^{18}$, low driving fields $s^{34}$, a random distribution of pinning centers ${ }^{20}$, and relatively small domain wall displacements ${ }^{18}$ ), the detailed mechanisms through which nanoscopic behavior (e.g. at a single domain wall) leads to Rayleigh behavior have remained mostly elusive. Physical descriptions of this phenomenological relationship have been offered since the works of $\mathrm{Née}^{35}$ and Kronmüller ${ }^{36}$, and though theoretical models of enhancements in piezoelectric coefficients from domain wall motion are considerable in scope, the experimental evidence at small length scales remained rather limited ${ }^{37,38}$. Detailed experimental investigations for ferroelectric capacitors were undertaken by Damjanovic and Trolier-McKinstry et al., beginning in the 1990's. Their research indicated that many polycrystalline ferroelectrics obey the Rayleigh relationships (for both piezoelectric and dielectric coefficients). It was shown that the extrinsic contributions to the dielectric and piezoelectric coefficients are consistent with irreversible domain wall motion in the sample, as confirmed by recent high-resolution in situ $\mathrm{x}$-ray diffraction studies ${ }^{39,40}$.

The Rayleigh relations provide the simplest mathematical framework to study the nonlinear dynamics in this system. To investigate the expected response from higher order harmonics, Fourier
Table 1 | Expected harmonic terms from the 'Dynamic Poling' model resulting from nearly reversible displacement of domain walls

\begin{tabular}{lll} 
& \multicolumn{1}{c}{ Linear in $\mathrm{E}$} & \multicolumn{1}{c}{ Quadratic in $\mathrm{E}$} \\
\hline $1^{\text {st }}$ order harmonic & $d_{0} E_{0} \sin (\omega t)$ & $\alpha_{D} E_{0}^{2} \sin (\omega t)+\frac{4 \alpha_{D} E_{0}^{2}}{3 \pi} \cos (\omega t)$ \\
$2^{\text {nd }}$ order harmonic & $\frac{\beta d_{0} E_{0}}{2} \cos (2 \omega t)$ & $\frac{\beta^{\prime} d_{0} E_{0}^{2}}{2} \cos (2 \omega t)$ \\
$3^{\text {rd }}$ order harmonic & 0 & $\frac{4 \alpha_{D} E_{0}^{2}}{15 \pi} \cos (3 \omega t)$ \\
\hline
\end{tabular}

expansion of the Rayleigh equation (2) can be carried out; this leads to only odd-order harmonic components that contribute to the strain $^{41}$. Furthermore, the third and higher order harmonic terms are characterized by a quadratic dependence of the strain on the driving field ${ }^{22}$. In many ferroelectrics, extrinsic contributions to the piezoelectric coefficients arise primarily from the motion of ferroelastic domain walls ${ }^{42,11}$. However, certain samples in which ferroelastic walls were not active still exhibited a linear dependence of the piezoelectric coefficient on the driving field ${ }^{43}$. To explain the discrepancy, the Rayleigh model was modified by Bassiri-Gharb et al. ${ }^{30}$ to include the effect of a domain wall (or any other interface) moving nearly-reversibly under applied fields in a 'dynamic poling' model. In such a situation, the $\mathrm{d}_{\text {init }}$, which was originally constant, becomes time and field-dependent, i.e. $d_{\text {init }}=d_{0}\left[1+\left(\beta+\beta^{\prime} E_{0}\right) \sin (\omega t)\right]$, reflective of a domain wall moving in-phase with the applied (sinusoidal) bias, and with the displacement linearly proportional to the applied field. Substituting this into equation 2, and developing the Fourier expansion ${ }^{30}$ produces the higher order harmonics and their field dependencies, which are organized in the table above for the first three harmonics (Note: static terms have been neglected, as they are not measured). It should also be noted that an additional contribution to the second harmonic arises from electrostriction.

Note that a similar analysis to that given above can be performed for more complex cases that include electrostrictive and spontaneous contributions to the total strain ${ }^{31}$ (see Supplementary S1).

From Table 1, it is clear that measurement of harmonic terms independently will allow decoupling of contributions to the strain from reversible and irreversible domain wall motions, providing the intrinsic electrostrictive strain is separately accounted for. A determination of the $3^{\text {rd }}$ order harmonic term (expected to scale as $E^{2}$ ), will allow the contributions from the irreversible motions of the domain walls to be determined, while the electrostrictive-like contributions to the strain from the reversible domain wall motions (which will add

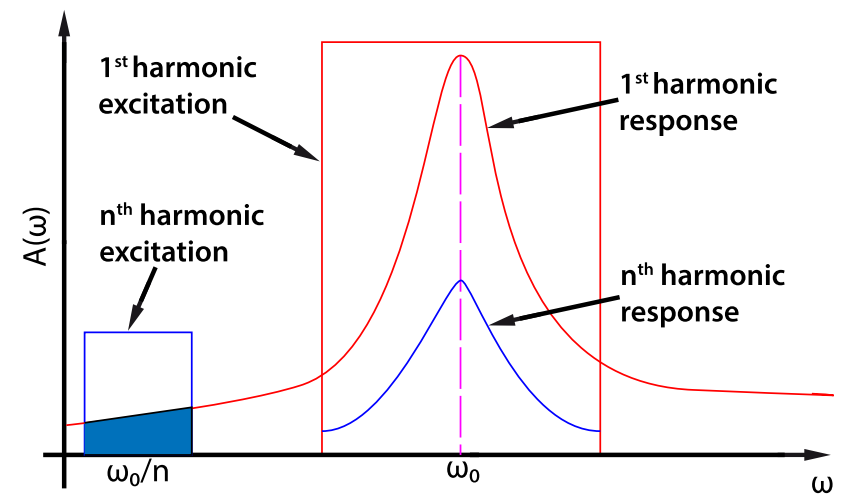

Figure $1 \mid$ General operating principle for nth harmonic detection. Using the band excitation method, a band of frequencies centered around $\omega_{0} / \mathrm{n}$ is excited, and the $\mathrm{n}^{\text {th }}$ harmonic response is (simultaneously) measured at the contact resonance $\omega_{0}$. 


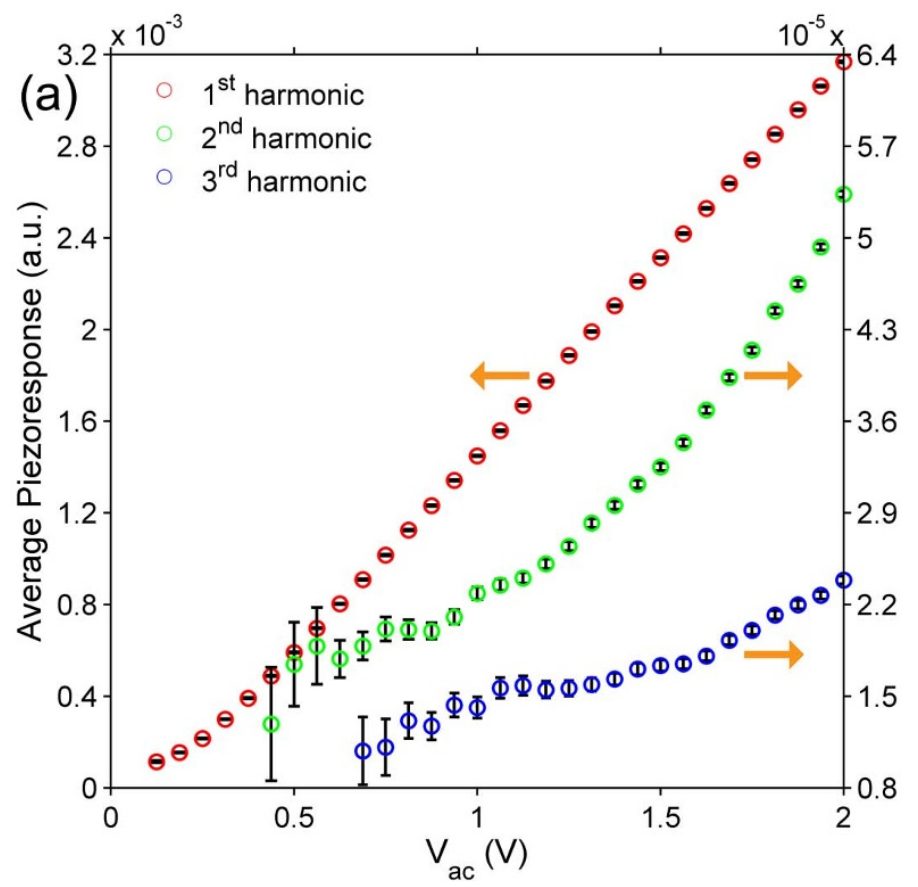

(b)

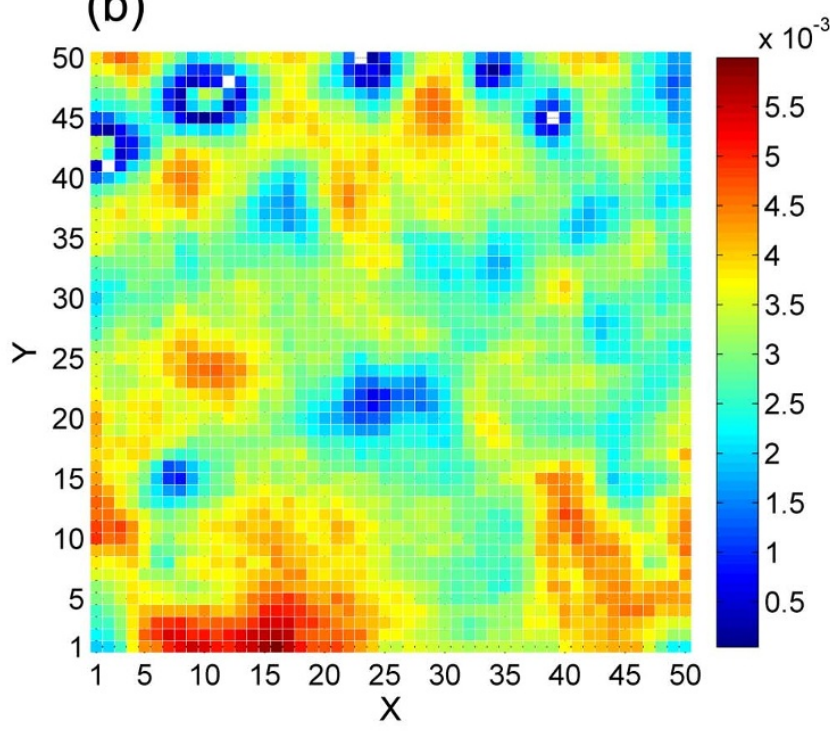

(c)
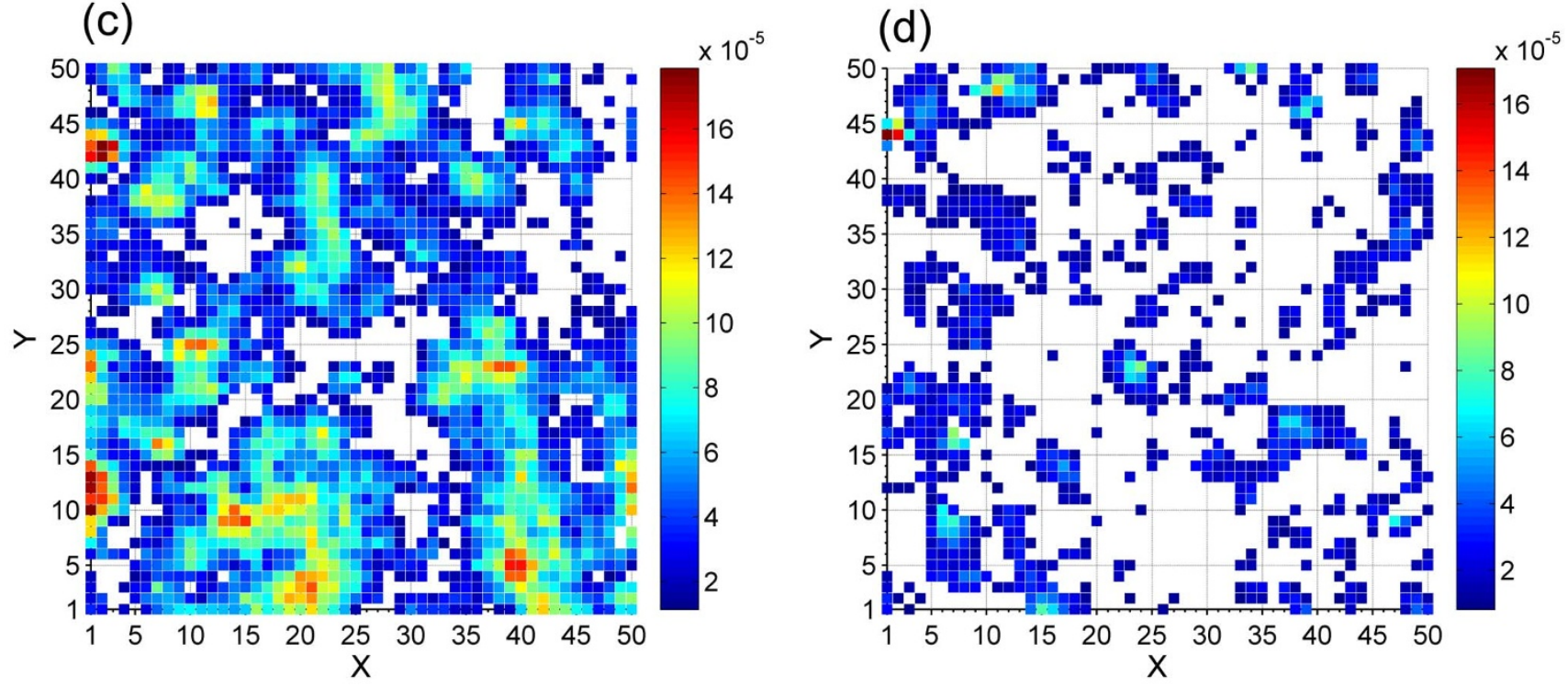

Figure $2 \mid$ Piezoresponse amplitude for $1^{\text {st }}, 2^{\text {nd }}$ and $3^{\text {rd }}$ harmonics, and mean response. The mean response for the three harmonics is shown in (a). The second and third harmonics are plotted on the right axis, and are two orders of magnitude weaker than the first. (b-d) The $50 \times 50$ grid $(5 \mu \mathrm{m} \times 5 \mu \mathrm{m})$ spatial maps of the piezoresponse amplitude (a.u.) at $\mathrm{V}_{\mathrm{ac}}=2 \mathrm{~V}$ are shown for (b) first, (c) second and (d) third harmonics. The white pixels in these spatial maps are points where a proper SHO fit was not possible.

to the real electrostrictive term) can be determined by calculating the $2^{\text {nd }}$ order harmonics. Furthermore, $\beta$ and $\beta$ ' can be separated if the field-dependencies of the response are measured. However, it is not yet clear whether such macroscopic models remain valid in the mesoscopic scale, and the length limits ${ }^{11}$ of these models have not been tested. In the next section, a technique to resolve these higher order harmonics, analyze the field dependencies, and correlate them locally at the nanoscale is discussed.

\section{Results}

The band-excitation (BE-) scanning probe microscopy technique for determining the first-order harmonic electromechanical response (piezoresponse) has been reported elsewhere ${ }^{44}$. Briefly, a band of frequencies is excited around the contact resonance of the cantilever (Figure 1, red square), and the response across the band is simultaneously measured and Fourier transformed to yield the system's total response. The BE-response is then fit to a simple harmonic oscillator (SHO) function to extract the response amplitude, phase, resonance and quality factor of the cantilever. The BEresponse is measured as a function of AC voltage. By judicious selection of the excitation function, it becomes possible to decouple the sample nonlinearity from the nonlinearity of the tip-surface junc$\operatorname{tion}^{45}$. This procedure is repeated across a grid of points on the sample, and the data can be fit to a predefined function at each point, to yield coefficient maps of the various fitting parameters. An extension to this technique (Figure 1) is the measurement of the $n^{\text {th }}$ harmonic ( $\mathrm{n}=1,2,3, \ldots$ ), where instead of exciting the band of frequencies at the contact resonance $\omega_{0}$, the excitation is around $\omega_{0} / n$. The measurement is still performed at the contact resonance; note that this technique has been demonstrated in a limited fashion 

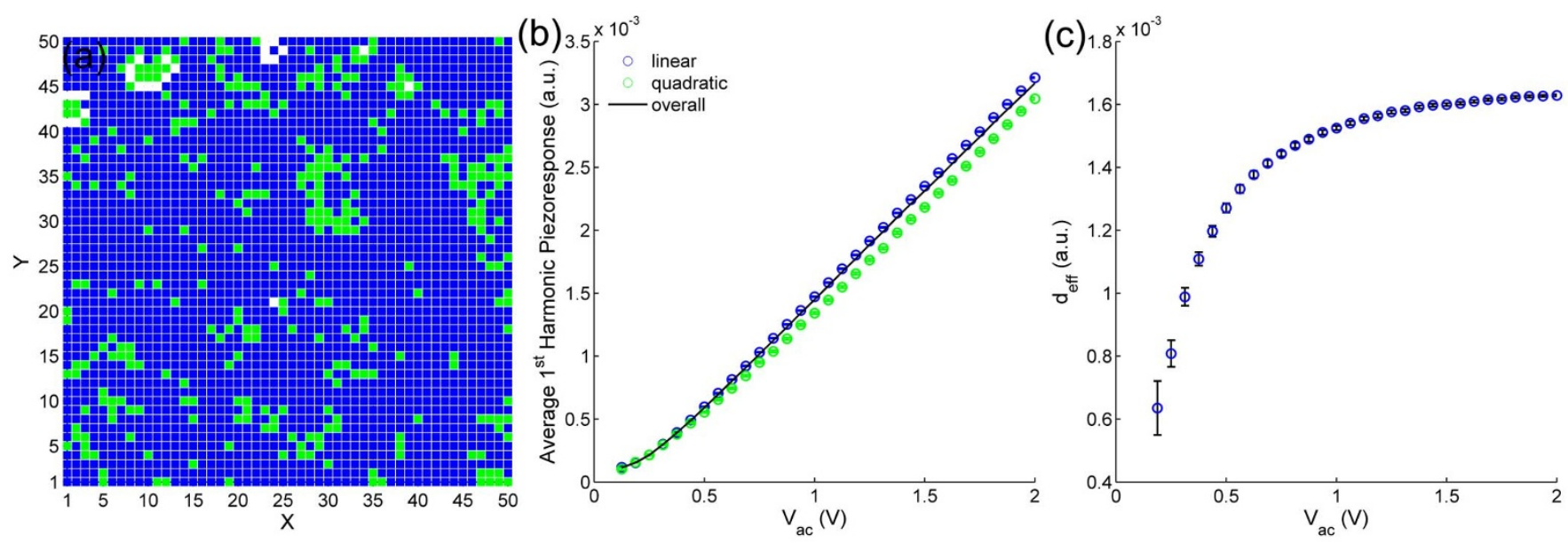

Figure $3 \mid$ Investigation of first harmonic response. (a) Fit-type map displaying regions which show linear, $\left(P=\alpha_{1} V_{a c}+c_{1}\right)$, (blue) and quadratic, $\left(P=\alpha_{1} V_{a c}^{2}+\alpha_{2} V_{a c}+c_{1}\right)$, (green) behaviors. (b) Average $1^{\text {st }}$ harmonic piezoresponse from blue and green points in (a) along with the overall average piezoresponse of the whole region inspected. (c) Effective piezoelectric coefficient $\mathrm{d}_{\mathrm{eff}}$.

for $n=2$ previously, though only for constant AC amplitude ${ }^{46}$. Measurement of higher order harmonics in this manner has two key advantages. First, there is a significant enhancement of the signal due to electromechanical coupling to the contact resonance of the cantilever; secondly, for higher order harmonics, if the excitation was at the contact resonance, then the detection would likely be in the $\mathrm{MHz}$ range, which is beyond the range of many SPM-based lock-in amplifiers.

The domain structure of the tetragonal PZT capacitor was initially explored through Piezoresponse Force Microscopy (PFM), see Supplementary S2. The sample is a $\sim 611 \mathrm{~nm}$ thick (001) tetragonal $\mathrm{Pb}\left(\mathrm{Zr}_{0.45}, \mathrm{Ti}_{0.55}\right) \mathrm{O}_{3}$ epitaxial film grown on $(001) \mathrm{SrTiO}_{3}$ substrate by pulsed laser deposition. X-ray diffraction indicated the sample is phase-pure. The film has a coercive field $\mathrm{E}_{\mathrm{c}}=\sim 44 \mathrm{kV} / \mathrm{cm}$.

The response of the first three harmonics was then investigated as a function of probing voltage $\left(\mathrm{V}_{\mathrm{ac}}\right)$, which in increments of $0.0625 \mathrm{~V}$ ranged from $\mathrm{V}_{\mathrm{ac}}=0.0625 \mathrm{~V}$ to $\mathrm{V}_{\mathrm{ac}}=2 \mathrm{~V}$ (corresponding to measurement up to $0.75 \mathrm{E}_{\mathrm{c}}$ ), on a $50 \times 50$ grid in a $5 \mu \mathrm{m} \times 5 \mu \mathrm{m}$ area yielding a spatial resolution of $100 \mathrm{~nm}$ per grid point. Maps of the piezoresponse and resonance as a function of $\mathrm{V}_{\mathrm{ac}}$ for all three harmonics are provided in Supplementary S3 and are also included as videos. The average response as a function of the applied voltage, for all three harmonics is plotted in Fig. 2(a). Error bars are plotted in black. The first harmonic signal appears largely linear, whereas the second and third harmonics can be better described by higher order polynomial functions. It is clear that the second and third harmonics of the piezoresponse are two orders of magnitude smaller than the first harmonic (Supplementary S4).

The voltage slices for the first piezoresponse of the three harmonics, i.e. the spatial maps, are shown for $\mathrm{V}_{\mathrm{ac}}=2 \mathrm{~V}$ in Fig. 2(b-d). The spatial maps for the resonance are given in Supplementary S5. The white pixels in these spatial maps are points where the response is close to zero, and thus were not amenable to fitting. The third harmonic in particular shows only a small number of spatial points that exhibit a measurable response. Interestingly, these tend to group in clusters. The second harmonic spatial map appears to show some correlation with the regions of high response in the first harmonic spatial map. The dynamic poling model, coupled with the observation that the second harmonic is spatially inhomogeneous, suggests that a portion of the second harmonic could be due to nearly reversible domain wall motion. That is, if the high local linear piezoelectric coefficient is enhanced by the existence of a contribution from nearly reversible domain wall motion, then it is reasonable that the second harmonic signal is high in the same regions of the sample.
Spatial correlations with the third harmonic and the other two harmonic response maps, however, are not evident.

In order to study the local responses, i.e. piezoresponse amplitude at a particular $(\mathrm{X}, \mathrm{Y})$ coordinate, in more detail, the dependence of the first harmonic piezoresponse amplitude on the probing voltage $\left(\mathrm{V}_{\mathrm{ac}}\right)$ was investigated. The local response was found to be a linear function of $\mathrm{V}_{\mathrm{AC}}\left(P=\alpha_{1} V_{a c}+c_{1}\right)$ in some regions, and a quadratic function of $\mathrm{V}_{\mathrm{ac}}\left(P=\alpha_{1} V_{a c}^{2}+\alpha_{2} V_{a c}+c_{1}\right)$ in others. A 'fit-type' map based on these two model response types considered shows these regions in Fig. 3(a). The majority of the studied area is linear (blue), but small clusters exhibiting quadratic behavior (green) also exist. However, the nonlinearity is relatively small for this film, suggesting that any domain walls that are contributing to the signal are doing so primarily in a reversible manner. The average response from the linear and quadratic regions is plotted in Fig. 3(b). The relatively small nonlinearity observed in this film may be the cause of the weak $3^{\text {rd }}$ harmonic signal, which should be locally correlated with the irreversible Rayleigh coefficient (Table 1). The effective piezoelectric coefficient $d_{\text {eff }}$ shown in Fig. 3(c), was calculated from the expression,

$$
d_{e f f}=\frac{\Delta P}{\Delta V_{a c}}=\frac{P\left(V_{a c}\right)-P\left(V_{r}\right)}{V_{a c}-V_{r}},
$$

where $\Delta \mathrm{P}$ and $\Delta \mathrm{V}_{\mathrm{ac}}$ are, respectively, the change in the overall average piezoresponse and $\mathrm{AC}$ probing voltage from the reference condition $\left(\mathrm{P}\left(\mathrm{V}_{\mathrm{r}}\right), \mathrm{V}_{\mathrm{r}}\right)$ where $\mathrm{V}_{\mathrm{r}}=0.0625 \mathrm{~V}$. The plot of $\mathrm{d}_{\text {eff }} \mathrm{vs}$. probing field reveals that for $\mathrm{V}_{\mathrm{ac}}<0.6 \mathrm{~V}$ (corresponding to $\mathrm{V}_{\mathrm{ac}}<0.22 \mathrm{E}_{\mathrm{c}}$ ), the $\mathrm{d}_{\text {eff }}$ is changing linearly with respect to applied field, suggesting that the measurements are within the Rayleigh regime.

\section{Discussion}

A first attempt was made to use the dynamic poling model to describe the quadratic function of the piezoelectric responses. It was found that since the majority of the data for higher harmonics were taken at voltages that exceed the Rayleigh regime, the results were not acceptable, i.e. the quality of the power-law fitting was such that the analytical model cannot be considered to be descriptive. Additional features in the local response at a particular $(\mathrm{X}, \mathrm{Y})$ coordinate shown in Fig. 4(a) appear very different from the average second harmonic response shown in Fig. 2(a). This type of response is clearly not amenable to quadratic fits (unlike the average second harmonic response), and it is comprised of a jagged response which we refer to as 'fine structure' overlaid on a monotonic increase. It is noted here that these fine structure features are found at nearly every point in the $(\mathrm{X}, \mathrm{Y})$ spatial map for the second harmonic, not just at this particular 

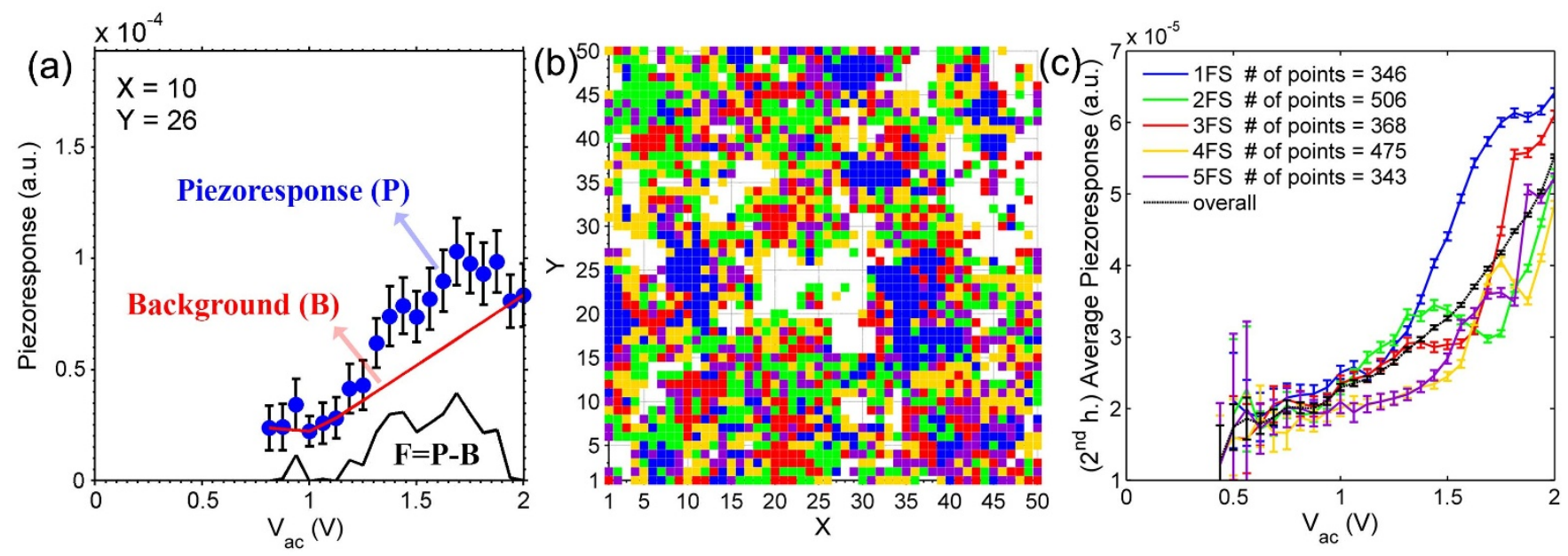

Figure $4 \mid$ Fine-structure analysis in $2^{\text {nd }}$ harmonic. An example of how the 'Fine Structure' (FS) in the $2^{\text {nd }}$ harmonic piezoresponse at a single (X,Y) point was obtained is shown in (a). The error bars shown correspond to $99 \%$ confidence intervals of the data points. The difference between the raw piezoresponse (P) and the background (B) constructed gives the FS data (black line, F=P-B). Subsequent analysis as explained in the text allows construction of an FS mask (b), which shows the most dominant eigenvector $w_{\mathrm{i}}$ (i.e. fine structure feature) at each $(\mathrm{X}, \mathrm{Y})$ coordinate in the studied region. Color code employed: blue, green, red, yellow and purple, respectively, for $w_{1}$ (i.e. 1FS) to $w_{5}$ (i.e. 5FS). (c) Average $2^{\text {nd }}$ harmonic response for the different colored regions in (b) with the overall average piezoresponse of the whole region inspected. The number of points stands for the total number of points populating the corresponding region in FS mask shown in (b).

point. Therefore, to study these fine structure features in more detail, a background was constructed using the bottom half segment of the convex hull of the data points to represent the monotonically increasing trend as shown by the red line in Fig. 4(a). (Supplementary S6). This background (B) was calculated for the second harmonic response at each $(\mathrm{X}, \mathrm{Y})$ position, and was subtracted from the raw second harmonic piezoresponse $(\mathrm{P})$ to produce a Fine Structure matrix $F$, i.e. $F=F\left(X, Y, V_{a c}\right)$. Here, the aim is to capture the portion of the data that represents deviation from a monotonically increasing trend, i.e. the convex hull of the data points. Principal Component Analysis (PCA) ${ }^{47}$, a convenient technique for visualizing trends in multidimensional datasets, was then performed on $F$, yielding a set of $N$ eigenvectors $w_{\mathrm{i}}\left(V_{a c}\right)$ with associated eigenvalues $\alpha_{i}(X, Y)$ which allows the dataset $F$ to be expressed as $F\left(X, Y, V_{a c}\right)=\sum_{i}^{N} \alpha_{i}(X, Y) w_{i}\left(V_{a c}\right)$. PCA is a decomposition, carried out in such a way that the first eigenvector (principal component) $w_{1}$ accounts for the highest statistical variation in the dataset, the second component $w_{2}$ accounts for the highest variation with the constraint it be orthogonal to the first eigenvector, and so on until the entire dataset can be described from the new set of eigenvectors. The corresponding loadings (eigenvalues) can be plotted spatially. For the PCA on $F$ (Supplementary S7), the first eigenvector $w_{1}$ contains one peak and corresponds to exactly one fine structure feature (1FS) appearing in the second harmonic response, the second eigenvector $w_{2}$ contains one peak and one valley and corresponds to two features (2FS), and so on for the first five eigenvectors (higher order eigenvectors are dominated by noise). The number of fine structure features is equal to the total number of peaks and valleys present in the eigenvector. The data $F\left(X, Y, V_{a c}\right)$ obtained at each point $(\mathrm{X}, \mathrm{Y})$ can be associated with the eigenvector $w_{\mathrm{i}}$ for which the eigenvalue $a_{\mathrm{i}}$ is the largest one at that point (i.e., the largest projection). In this manner, fine structure features (1FS to 5FS) can be determined for each point $(\mathrm{X}, \mathrm{Y})$ and plotted spatially to obtain an FS mask (or regional deconstruction) as shown in Fig. 4(b).

Interestingly, this regional deconstruction shows that the fine structure features tend to cluster, and is particularly evident for points which display one (blue), two (green) and three (red) fine structure features. The associated plots of the average second harmonic response from each of these colored regions are given in Fig. 4(c) along with their overall average response. More information on the local cluster behavior can be found in Supplementary S8. Surprisingly, averaging of all the local responses leads to an overall average response [solid black line in Fig. 4(c)] that can be better approximated by a polynomial function, in stark contrast to the local response. However, the sum average of the second harmonic appears to be much more closely aligned with the expected macroscopic response, indicating that the fine structures are in fact arising from local, frozen disorder in the material.

In summary, a generalized scanning probe microscopy technique to explore nonlinear dynamics, through $\mathrm{n}^{\text {th }}$-order harmonic detection with nanometer spatial resolution is presented. For the first time, the appearance of fine structure features in the second harmonic are identified and suggested to arise from local frozen disorder. The novel technique used here potentially allows the length scales of macroscopic theories to be tested, and furthermore sheds light on how properties develop at the nanoscale. This study reveals the potential of harmonic detection in uncovering nonlinear dynamics in material systems.

\section{Methods}

The $\mathrm{n}^{\text {th }}$ harmonic detection system was implemented on a commercially available AFM platform (Cypher Model, Asylum Research) equipped with in-house bandexcitation performed with National Instruments PXI-based electronics with Labview and Matlab-based scripts. The sample is a $\sim 611 \mathrm{~nm}$ thick (001) tetragonal $\mathrm{Pb}\left(\mathrm{Zr}_{0.45}, \mathrm{Ti}_{0.55}\right) \mathrm{O}_{3}$ epitaxial film grown on (001) $\mathrm{SrTiO}_{3}$ substrate by pulsed laser deposition. X-ray diffraction indicated the sample is phase-pure. The film has a coercive field $\mathrm{E}_{\mathrm{c}}=\sim 44 \mathrm{kV} / \mathrm{cm}$. Numerical analysis was performed with Matlab v7.

1. Boyd, R. W. Nonlinear Optics. (Academic Press, 2008).

2. Silberberg, M. S. Chemistry: The Molecular Nature of Matter and Change. (McGraw-Hill Higher Education, 2008).

3. Parkin, S. S. P., Hayashi, M. \& Thomas, L. Magnetic Domain-Wall Racetrack Memory. Science 320, 190-194 (2008).

4. Scott, J. F. \& Paz de Araujo, C. A. Ferroelectric Memories. Science 246, 1400-1405 (1989).

5. Dubi, Y., Meir, Y. \& Avishai, Y. Nature of the superconductor-insulator transition in disordered superconductors. Nature 449, 876-880 (2007).

6. Kutnjak, Z., Petzelt, J. \& Blinc, R. The giant electromechanical response in ferroelectric relaxors as a critical phenomenon. Nature 441, 956-959 (2006).

7. Keys, A. S., Abate, A. R., Glotzer, S. C. \& Durian, D. J. Measurement of growing dynamical length scales and prediction of the jamming transition in a granular material. Nat. Phys. 3, 260-264 (2007).

8. Hamann, C. H., Hamnett, A. \& Vielstich, W. Electrochemistry. (Wiley-VCH, 2007). 
9. Fiebig, M., Pavlov, V. V. \& Pisarev, R. V. Second-harmonic generation as a tool for studying electronic and magnetic structures of crystals: review. J. Opt. Soc. Am. B 22, 96-118 (2005)

10. Martin, Y., Abraham, D. W. \& Wickramasinghe, H. K. High-resolution capacitance measurement and potentiometry by force microscopy. Appl. Phys. Lett. 52, 1103-1105 (1988)

11. Vasudevan, R. K. et al. Nanoscale origins of nonlinear behavior in ferroic thin films. Adv. Func. Mat. 23, 81-90 (2013).

12. Wilson, J. R., Sase, M., Kawada, T. \& Adler, S. B. Measurement of Oxygen Exchange Kinetics on Thin-Film $\mathrm{La}_{0.6} \mathrm{Sr}_{0.4} \mathrm{CoO}_{3-\delta}$ Using Nonlinear Electrochemical Impedance Spectroscopy. Electrochemical and Solid-State Letters 10, B81-B86 (2007).

13. Wilson, J. R., Schwartz, D. T. \& Adler, S. B. Nonlinear electrochemical impedance spectroscopy for solid oxide fuel cell cathode materials. Electrochimica Acta 51, 1389-1402 (2006).

14. Seeger, K. \& Maurer, W. Nonlinear electronic transport in TTF-TCNQ observed by microwave harmonic mixing. Solid State Commun. 27, 603-606 (1978).

15. Lehmann, W. et al. Giant lateral electrostriction in ferroelectric liquid-crystalline elastomers. Nature 410, 447-450 (2001).

16. Turik, A. Theory of polarization and hysteresis of ferroelectrics. Sov Phys-Sol State 5, 885-886 (1963).

17. Boser, O. Electromechanical resonances in ceramic capacitors and evaluation of the piezoelectric materials' properties. Advanced Ceramic Materials 2, 167-172 (1987).

18. Boser, O. Statistical theory of hysteresis in ferroelectric materials. J. Appl. Phys. 62, 1344-1348 (1987).

19. Kronmüller, H. \& Angew, Z. Statistical Theory of Rayleigh's Law. Physik 30, 9 (1970).

20. Damjanovic, D. Stress and frequency dependence of the direct piezoelectric effect in ferroelectric ceramics. J. Appl. Phys. 82, 1788-1797 (1997).

21. Damjanovic, D. Logarithmic frequency dependence of the piezoelectric effect due to pinning of ferroelectric-ferroelastic domain walls. Phys. Rev. B. 55, R649 (1997).

22. Damjanovic, D. in Science of Hysteresis Vol. 3 (eds Giorgio. Bertotti \& Isaak D. Mayergoyz) 337-465 (Elsevier Inc., 2005).

23. Xu, F., Chu, F. \& Trolier-McKinstry, S. Longitudinal piezoelectric coefficient measurement for bulk ceramics and thin films using pneumatic pressure rig. J. Appl. Phys. 86, 588-594 (1999).

24. Xu, F. et al. Domain wall motion and its contribution to the dielectric and piezoelectric properties of lead zirconate titanate films. J. Appl. Phys. 89, 1336-1348 (2001)

25. Leary, S. P. \& Pilgrim, S. M. Harmonic analysis of the polarization response in $\mathrm{Pb}\left(\mathrm{Mg}_{1 / 3} \mathrm{Nb}_{2 / 3}\right) \mathrm{O}_{3}$-based ceramics-A study in aging. IEEE T. Ultrason. Ferr. $\mathbf{4 5}$ 163-169 (1998).

26. Sherlock, N. \& Meyer, R. Large signal response and harmonic distortion in piezoelectrics for SONAR transducers. J. Electroceram. 28, 202-207 (2012).

27. Hemberger, J., Lunkenheimer, P., Viana, R., Böhmer, R. \& Loidl, A. Electric-fielddependent dielectric constant and nonlinear susceptibility in $\mathrm{SrTiO}_{3}$. Phys. Rev. B. 52, 13159-13162 (1995).

28. Glazounov, A. E. \& Tagantsev, A. K. Phenomenological Model of Dynamic Nonlinear Response of Relaxor Ferroelectrics. Phys. Rev. Lett. 85, 2192-2195 (2000).

29. Gharb, N. B., Trolier-McKinstry, S. \& Damjanovic, D. Piezoelectric nonlinearity in ferroelectric thin films. J. Appl. Phys. 100, 044107 (2006).

30. Trolier-McKinstry, S., Gharb, N. B. \& Damjanovic, D. Piezoelectric nonlinearity due to motion of $180^{\circ}$ domain walls in ferroelectric materials at subcoercive fields: A dynamic poling model. Appl. Phys. Lett. 88, 202901 (2006).

31. Bassiri-Gharb, N., Trolier-McKinstry, S. \& Damjanovic, D. Strain-modulated piezoelectric and electrostrictive nonlinearity in ferroelectric thin films without active ferroelastic domain walls. J. Appl. Phys. 110, 124104 (2011).

32. Karami, M. A. \& Inman, D. J. Powering pacemakers from heartbeat vibrations using linear and nonlinear energy harvesters. Appl. Phys. Lett. 100, 042901 (2012).

33. Park, S. E. \& Shrout, T. R. Relaxor based ferroelectric single crystals for electromechanical actuators. Materials Research Innovations 1, 20-25 (1997).

34. Hall, D. A. Review Nonlinearity in piezoelectric ceramics. J. Mater. Sci. 36, 4575-4601 (2001).

35. Néel, L. Theory of Rayleigh's Laws of magnetization. Cahiers Phys. 12 (1942).

36. Kronmüller, H. Theory of Rayleigh'zs law in magnetically multiaxial and uniaxial crystals. J. Phys. Colloque C1 32, 390 (1971).
37. Nattermann, T., Shapir, Y. \& Vilfan, I. Interface pinning and dynamics in random systems. Phys. Rev. B. 42, 8577-8586 (1990).

38. Nattermann, T. Interface roughening in systems with quenched random impurities. EPL (Europhysics Letters) 4, 1241-1246 (1987).

39. Pramanick, A., Damjanovic, D., Nino, J. C. \& Jones, J. L. Subcoercive cyclic electrical loading of lead zirconate titanate ceramics I: nonlinearities and losses in the converse piezoelectric effect. J. Am. Cer. Soc. 92, 2291-2299 (2009).

40. Pramanick, A., Damjanovic, D., Daniels, J. E., Nino, J. C. \& Jones, J. L. Origins of electro-mechanical coupling in polycrystalline ferroelectrics during subcoercive electrical loading. J. Am. Cer. Soc. 94, 293-309 (2011).

41. Rayleigh, L. XXV. Notes on electricity and magnetism.-III. On the behaviour of iron and steel under the operation of feeble magnetic forces. Philos. Mag. Ser. 23, 225-245 (1887)

42. Pertsev, N. \& Arlt, G. Forced translational vibrations of $90^{\circ}$ domain walls and the dielectric dispersion in ferroelectric ceramics. J. Appl. Phys. 74, 4105-4112 (1993).

43. Gerber, P., Kugeler, C., Bottger, U. \& Waser, R. Effects of ferroelectric switching on the piezoelectric small-signal response $\left(\mathrm{d}_{33}\right)$ and electrostriction $\left(\mathrm{M}_{33}\right)$ of lead zirconate titanate thin films. J. Appl. Phys. 95, 4976-4980 (2004).

44. Bintachitt, P. et al. Collective dynamics underpins Rayleigh behavior in disordered polycrystalline ferroelectrics. Proc. Nat. Acad. Sci. USA 107, 7219-7224 (2010).

45. Griggio, F. et al. Mapping piezoelectric nonlinearity in the Rayleigh regime using band excitation piezoresponse force microscopy. Appl. Phys. Lett. 98, 212901 (2011).

46. Kim, Y. et al. Nonlinear Phenomena in Multiferroic Nanocapacitors: Joule Heating and Electromechanical Effects. ACS Nano 5, 9104-9112, http:// dx.doi.org/10.1021/nn203342v (2011).

47. Jesse, S. \& Kalinin, S. V. Principal component and spatial correlation analysis of spectroscopic-imaging data in scanning probe microscopy. Nanotechnology 20, 085714 (2009).

\section{Acknowledgements}

STM and DM gratefully acknowledge support from the National Science Foundation (DMR 1005771) and a National Security Science and Engineering Faculty Fellowship. R.K.V. M.B.O. and N.V. acknowledge support from the ARC Discovery Project scheme. R.K.V. and V.N. acknowledge an Overseas Travel Fellowship by the Australian Nanotechnology Network and the user facilities at ORNL-CNMS under user proposal No. 2011-281. The research at ORNL (Y.K., S.J., S.V.K.) was conducted at the Center for Nanophase Materials Sciences, which is sponsored at Oak Ridge National Laboratory by the Division of Scientific User Facilities, U.S. Department of Energy.

\section{Author contributions}

R.K.V. performed the experiments, analyzed data, prepared figures and wrote the paper. M.B.O. analyzed the data, wrote the Matlab scripts for data analysis and fitting, prepared figures and co-wrote the paper. R.K.V. and M.B.O. contributed equally to this manuscript. I.R., Y.K. and S.J. helped perform the experiments, developed and implemented the $\mathrm{n}^{\text {th }}$ harmonic excitation technique on the Asylum platform. D.M. prepared the sample used for study, analyzed data and performed relevant characterization experiments, and co-wrote the paper. S.T.M., V.N. and S.V.K. planned the study, analyzed the data and co-wrote the paper. All authors reviewed the manuscript.

\section{Additional information}

Supplementary information accompanies this paper at http://www.nature.com/ scientificreports

Competing financial interests: The authors declare no competing financial interests How to cite this article: Vasudevan, R.K. et al. Higher order harmonic detection for exploring nonlinear interactions with nanoscale resolution. Sci. Rep. 3, 2677; DOI:10.1038/ srep02677 (2013)

(c) (i) $\Theta$ This work is licensed under a Creative Commons Attributionvisit http://creativecommons.org/licenses/by-nc-nd/3.0 